\title{
Fast Analysis of Intracranical Aneurysms Based on Interactive Direct Volume Rendering and CTA
}

\author{
P. Hastreiter ${ }^{1}$, Ch. Rezk-Salama ${ }^{1}$, B. Tomandl ${ }^{2}$, K.E.W. Eberhardt ${ }^{2}$, T. Ert ${ }^{1}$ \\ 1 Computer Graphics Group, University of Erlangen-Nuremberg, \\ Department of Computer Science, Am Weichselgarten 9, 91058 Erlangen, Germany \\ \{hastreiter|ert 1 \} informatik.uni-erlangen.de \\ 2 Division of Neuroradiology, University of Erlangen-Nuremberg, \\ Department of Neurosurgery, Schwabachanlage 6, 91054 Erlangen, Germany
}

\begin{abstract}
The diagnosis of intracranial aneurysms and the planning of related interventions is effectively assisted by spiral CT-angiography and interactive direct volume rendering. Based on 3D texture mapping, we suggest a hardware accelerated approach which provides fast and meaningful visualization without time-consuming pre-processing. Interactive tools provide reliable measurement of distance and volume allowing to calculate the size of vessels and aneurysms directly within the 3D viewer. Thereby, the expensive material required for coiling procedures is estimated more precisely. Interactively calculated shaded isosurfaces, presented in [1] were evaluated in respect of enhanced perception of depth. Based on the integration into OpenInventor, global overview and simultaneous detail information is provided by communicating windows allowing for intuitive and user-guided navigation. Due to an average of 15-20 minutes required for the complete medical analysis, our approach is expected to be useful for clinical routine. Additional registration and simultaneous visualization of MR and CT-angiography gives further anatomical orientation. Several examples demonstrate the potential of our approach.
\end{abstract}

\section{Introduction}

Current clinical routine is supported by different imaging modalities which allow to perform angiographic procedures. They provide important information for the diagnosis of a diversity of vessel pathologies. DSA (Digital Subtraction Angiography) is the most established imaging technique for cerebral blood vessels and their malformations. Although there is a certain risk of complications due to its invasive nature, DSA is the method of choice for cerebral aneurysms providing optimal spatial and temporal resolution. However, in case of complex topology the superposition of vessels makes the analysis difficult since the resulting projection images contain little depth information (see Fig. 1). Therefore, tomographic imaging techniques like CTA (Computed Tomography Angiography) and MRA (Magnetic Resonance Angiography) gain growing attention since they provide three-dimensional $(3 D)$ information.

Intracranial aneurysms are berry-like blisters of the cerebral arteries which are caused by a weakness of all vessel wall layers. They incidentally occur in 1 to 5 out of 100 individuals [2] as congenital lesion or after a disease of the vessel wall. As a major 
risk, hemorrhage may cause serious damage to the brain, if the rupture of an aneurysm occurs. In preparation of interventional procedures or surgery a clear understanding of the surrounding vessel situation is required. The location and size of the neck which is the connection between the aneurysm and the feeding vessel, strongly influences the further strategy. In general, there are two methods of treatment, with surgery performed in most cases. More recently, an alternative procedure allows to direct a platinum coil into the aneurysm using an intra-vascular access. In order to perform a fast and precise analysis the choice of the imaging modality combined with an appropriate visualization approach are of great importance.

Aiming at 3D imaging modalities, aneurysms with a diameter of $3-11 \mathrm{~mm}$ are reproduced with similar quality. However, in case of MRA it is still difficult to delineate the neck and small arterial branches due to the nature of the resulting images. Above all, a meaningful visualization requires several hours of pre-processing, as presented in [3]. Therefore, as proposed in [4], we use CTA which provides the highest sensitivity for lesions of arbitrary size including small $(>3 \mathrm{~mm})$ and large $(>11 \mathrm{~mm})$ aneurysms.

Since it is difficult to integrate the information of tomographic slice images to a correct 3D model, reconstruction is efficiently assisted by techniques of volume rendering. For the analysis of angiographic data sets maximum intensity projection has positioned itself as one of the most popular visualization techniques. However, using only the highest gray value along every ray of sight, it prohibits to distinguish overlapping vessels clearly and to see intra-vascular abnormalities. Alternatively, techniques of surface rendering became very popular $[3,5]$ which allow to compute images interactively based on an intermediate geometric model. However, its calculation requires time-intensive segmentation which must be repeated for every correction of the result.

In order to overcome these limitations, we suggest direct volume rendering which uses the entire data volume, giving insight to interior and superimposed information. In order to provide interactive manipulation of the visualization parameters which is essential for the analysis in practice [6], we propose to use hardware accelerated 3D texture mapping [7] which we expect to be commonly available on PCs in the near future. After a short survey of our visualization approach in section 2, we briefly review the interactive calculation of iso-value representations in section 3 which has been previously published in [1]. Their integration was evaluated for the diagnosis of cerebral aneurysms in respect of enhanced perception of depth. Subsequently, in section 4, we suggest intuitive manipulation based on the integration into OpenInventor. In sections 5 and 6 tools are presented which consider convenient measurement of aneurysms and the registration of MR and CT based on a hardware accelerated approach, which was introduced in [8]. Finally, the strategy of the medical diagnosis is presented in section 7 with several examples demonstrating the value of our approach.

\section{Direct Volume Rendering}

For the visualization of tomographic image data, direct volume rendering proved to be very suitable. It allows to apply user-defined transfer functions which assign a specific color and opacity to all voxels of a certain scalar value. According to [9], all known approaches of direct volume rendering can be reduced to the transport theory model 
which describes the propagation of light in materials. Approximations of the underlying equation of light transfer have been developed and differ considerably in the physical phenomena they account for and in the way the emerging numerical models are solved.

The large amount of data of a typical tomographic scan makes the interactive manipulation of the volume object difficult which is essential for the ease of interpretation and the convenient analysis in medical practice. Therefore, based on ray-casting different optimization techniques were proposed $[10,11]$ which allow to traverse the volume data more efficiently. Alternatively, the time-intensive re-sampling of the original data is circumvented by voxel based projection methods [12]. More recently, considerable acceleration was achieved by expressing the rotation of a volume object by a $2 \mathrm{D}$ shear and a consecutive image warp [13]. However, most impressive frame rates are obtained with special purpose hardware $[14,15]$.

The 3D texture mapping hardware of high-end graphics computers provides a huge amount of trilinear interpolation operations and thereby allows to perform direct volume rendering at high image quality and interactive frame rates [7]. After converting the volume data set to a $3 \mathrm{D}$ texture, it is loaded to the respective memory. In a first step equidistant planes parallel to the viewport are clipped against the bounding box of the volume data set. Subsequently, the resulting polygons are projected onto the image plane. During rasterization they are textured with their corresponding image information directly obtained from the 3D texture by trilinear interpolation. Finally, the $3 \mathrm{D}$ representation is produced by successive blending of the textured polygons backto-front onto the viewing plane. Since this process uses the blending and interpolation capabilities of the underlying hardware, the time consumed for the generation of an image is negligible compared to software based approaches. Interactive frame rates can be achieved even when applied to data sets of high resolution.

\section{Fast Iso-Surfaces}

Semi-transparent representations produced with direct volume rendering techniques allow to see superimposed structures. However, for some directions of view the spatial relation is evaluated much easier if lighting effects are integrated (see Fig. 2). If interactive navigation through complex vessel structures is envisaged, shaded surfaces assist to improve the users sense of orientation.

Different approaches like the Marching Cubes algorithm [16] provide iso-value representations of structures contained in volume data sets. However, as a major drawback, time-intensive calculations are required for every change of the iso-value prohibiting interactive manipulation. In addition, the complexity of the intermediate geometric model rapidly exceeds the available rendering capacity.

As presented in [1], interactive manipulation of iso-value surfaces and realistic lighting effects are possible if hardware assisted 3D texture mapping and two frame buffer operations are applied. This requires to define a cut plane for every scan line of the resulting image which has an orientation orthogonal to the viewing plane. Applying the rendering procedure, described in section 2, the corresponding image information of every cut plane is interpolated within the $3 \mathrm{D}$ texture memory and written to the frame buffer. After accessing its contents, all data values required for a complete scan line 
are simultaneously available in real-time. Using a standard ray-casting procedure they have to be calculated separately for every pixel of the current scan line along rays in the direction of view. After checking the obtained cut plane for the current iso-value the identified 2D locations are re-projected to their 3D positions within the volume data. Interpolation of the neighboring voxel gradients finally provides the surface normals required for the shading procedure.
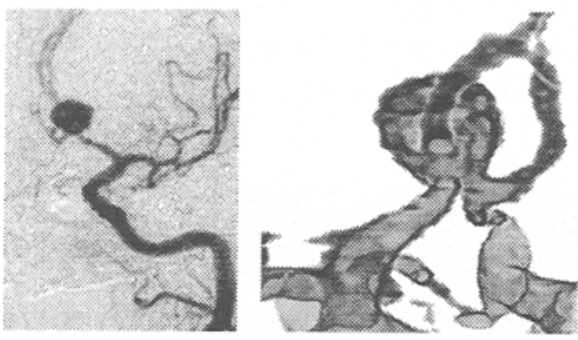

Fig. 1. Intracranial aneurysm: DSA (left) and direct volume rendering of CTA (right).

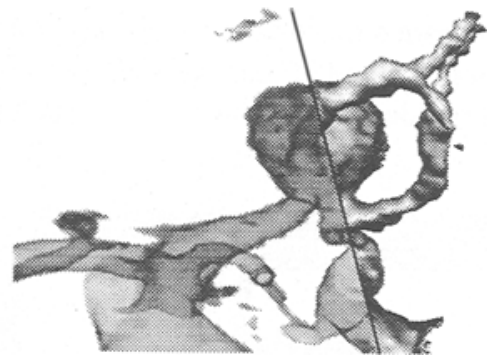

Fig. 2. Comparison of semi-transparent (left) and shaded iso-surface (right) representation.

\section{Manipulation and Navigation}

The presented approach builds on top of a frame work based on OpenInventor which was previously presented in [1]. Thereby, a variety of functionality is available which ensures intuitive manipulation. As a basis, a standardized interface is offered which provides a selection of sophisticated 3D viewers including various editors, light sources and other features. Additionally, several built-in manipulators are available which are attached to an object after selection. They considerably support a convenient navigation within 3D space if a 2D input device is applied. Consequently, the user-guided interactive inspection and analysis of an angiographic volume data set is accelerated.

In order to inspect interior structures, independent clip planes provide an intuitive way to virtually cut off parts of the volume data set. As can be seen in Fig. 4, the wall of the aneurysm was partly removed in order to view the connection to a related vessel from inside. Alternatively, choosing normal rendering mode and full opacity for the whole data set, clip planes assist the clinician to inspect the local gray value information at slices with arbitrary orientation.

In many situations during the analysis of an angiographic data set it is necessary to inspect detail information by approaching the structures very closely. However, at the same time, it is desirable to view the lesion from a more distant eye-point. Therefore, two communicating 3D displays are provided (see Fig. 3) which allow for an individual adjustment of the camera position. Within the right window typically local information is focused while a more distant view point gives global overview of the vessel situation within the left window. In order to provide improved orientation a geometric 
representation of the viewing frustum of the right window is integrated into the left display. Thereby, the small arrow connected to a sphere indicates the direction of view and the eye-point. Optionally, if the camera parameters of the right window change, the position of the camera of the left window remains unchanged, either relative to the arrow-sphere model or to the volume object. Thereby, the user gets the impression of a passing volume object in the first case, or of an arrow which flies through the fixed data set. Alternatively, if the arrow-sphere model is selected in the left window different manipulators are attached which allow to change the viewing parameters of the right window from a more distant viewing point. Finally, if the "fly-mode" option of OpenInventor is applied interactive and user-controlled navigation within vessels is possible which provides endoscopic views of the sac or the neck of an aneurysm.

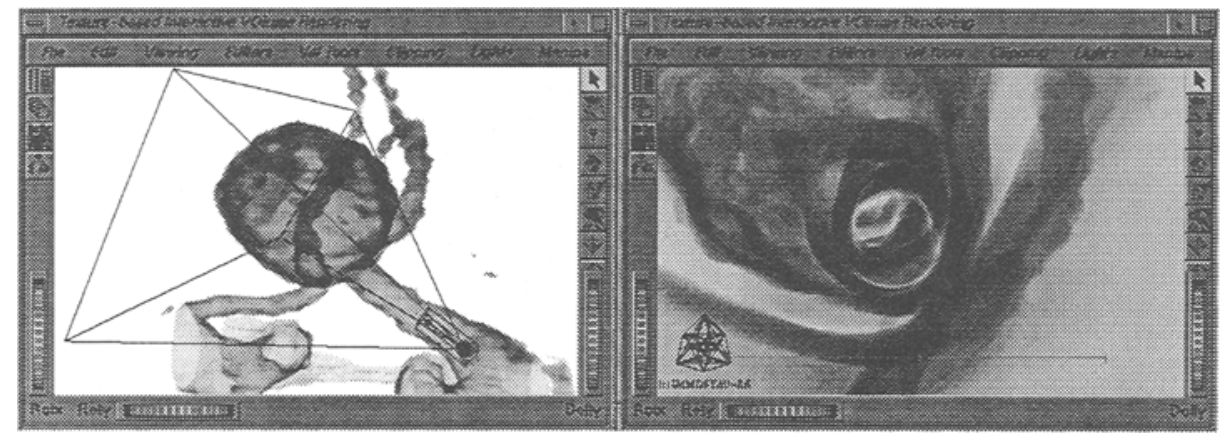

Fig. 3. Navigation inside a vessel approaching the aneurysm. The arrow within the left window indicates the direction of view of the right window

\section{Measurements}

For the preparation of interventions it is helpful to have information about the size of the aneurysm, the size of vessels or the distance between adjacent structures. Therefore, simple interactive tools are provided which allow for measurements directly within the 3D viewer (see Fig. 5). Thereby, the calculation of the distance between two points is performed on-the-fly, after placing the corresponding markers in 3D space. Finding the right position is assisted by appropriate manipulators inherited from the OpenInventor toolkit. But, more efficiently the correct location can be identified, if additional clip planes are used to reduce the degrees of freedom.

If treatment with a platinum coil is envisaged, additional knowledge about the volume of the aneurysm allows better estimation of the required expensive material. Using simple geometric objects the actual shape of an aneurysm or vessel is approximated. During an iterative procedure the shape of the selected object is distorted with an appropriate manipulator of OpenInventor while the quality of the fit is visually inspected by rotating the whole scene. 


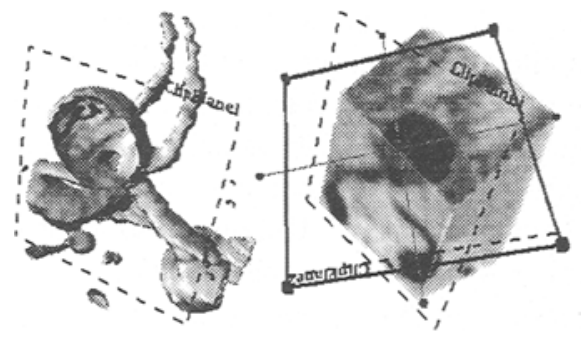

Fig. 4. Clip planes: inspect interior structures (left) and gray value information (right).

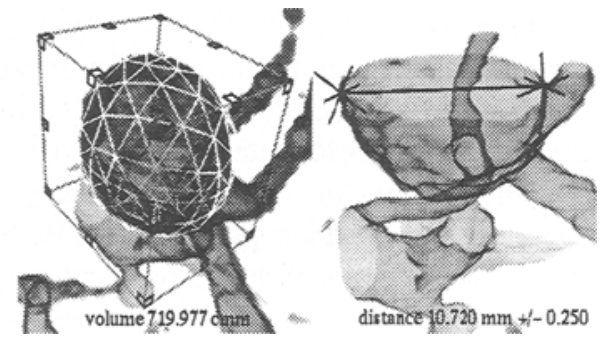

Fig. 5. On-the-fly measurement of volume (left) and distance (right).

\section{Registration}

In order to provide further anatomical orientation an additional tool is provided which allows for fast registration of the angiographic $\mathrm{CT}$ data with MR. As presented just recently [17], voxel based approaches gained more attention since they allow to perform retrospective and accurate alignment. Taking into account the entire gray value information at geometrically corresponding grid positions, the similarity of the involved data sets is optimized using a functional which evaluates the quality of alignment. Having introduced mutual information $[18,19]$, a very general functional is available which proved to provide very robust registration. However, the optimization procedure which is required to re-sample the volume data, typically consumes a huge amount of trilinear interpolation operations. Ensuring fast performance which is fundamental for medical applications, an approach is provided which integrates registration into the process of visualization, as suggested in [8]. Similar to the technique used for direct volume rendering the method uses the imaging and texture mapping subsystem of graphics computers. Thereby, all trilinear interpolation operations are completely performed with hardware assisted 3D texture mapping. The histograms of the data sets which are necessary for the calculation of mutual information are obtained with different hardware accelerated imaging operations. Using similar hardware supported procedures, as applied to visualization, the process of registration is considerably accelerated.

\section{Strategy of Data Analysis}

The visualization and inspection of CTA data sets is performed according to a specific protocol in order to ensure a fast and consistent analysis. After transferring the data from the scanner to a graphics workstation the range of gray values which describes the vessel information is transformed linearly to a new representation while data values below are suppressed and those above are mapped to maximum value. Due to the stretching of the histogram in the area of interest, subsequent manipulation of transfer functions for color and opacity values is performed in a more convenient way. Applying pre-defined lookup tables, a fast and meaningful initial visualization is obtained. Subsequently, the transfer functions are adjusted interactively to the individual data set by simple manipulation operations while the result is reviewed by a skilled user. 
In the beginning, the whole data volume is loaded in order to detect all aneurysms. Pre-defined eye points and directions of view allow for a fast access of the most important areas for the medical analysis. Subsequently, the user may rotate, translate or zoom the $3 \mathrm{D}$ representation interactively in order to inspect detail information. Following the step of identification, bounding boxes are applied which define a sub-volume around every aneurysm which was detected. Reducing the amount of data allows to focus on the essential details of the vessel topology and ensures real-time manipulation.

For the documentation of every case, snap shots of the current visualization can be taken at any time of the analysis. Additionally, short movie sequences are recorded in order to provide an enhanced perception of depth.

\section{Results and Discussion}

All image data was obtained with a Siemens Somaton Plus 4 spiral-CT scanner. For the angiography $100 \mathrm{ml}$ of non-ionic contrast agent were injected with a delay time dependent on the circulation time and a flow rate of $3 \mathrm{ml} / \mathrm{sec}$. With the lowest possible table-feed and a slice thickness of $1 \mathrm{~mm}$, volume data sets with a resolution of $0.2 \times 0.2$ $\mathrm{x} 0.5 \mathrm{~mm}$ were reconstructed. The applied imaging technique proved to be optimal for small vessels and aneurysms including those of the basilar artery. All visualization was performed on a SGI Indigo ${ }^{2}$ Maximum Impact and a SGI Onyx Reality Engine II which provide 4 Mbytes and 16 Mbytes of 3D texture memory respectively. Concerning the amount of available trilinear interpolation operations the underlying hardware has the same order of magnitude.

Subsequently, image data of three patients is presented which was obtained with the proposed approach. The overview image of Fig. 6 shows the location of a large basilar bifurcation aneurysm relative to the surrounding vessel situation. Choosing a closer semi-transparent representation the connections to the basilar artery and both posterior cerebral arteries are clearly conveyed. Within Fig. 7 an aneurysm of the right anterior communicating artery $(\mathrm{ACOA})$ is delineated. Intra-operative views (before and after applying a clip for treatment) demonstrate the similarity between real and virtual presentation. Finally, Fig. 8 shows an aneurysm of the middle cerebral artery (MCA) bifurcation. The close semi-transparent visualization of the aneurysm clearly depicts the connected vessels and their location relative to the lesion. (see the color page at: http://www9.informatik.uni-erlangen.de/eng/gallery/vis/med/miccai.html)

Our experiments have shown that the visualization method is of major importance for the diagnostic value of the obtained images. In comparison to exclusively using shaded surface representations, direct volume rendering has proved to be an optimal visualization approach in order to delineate the sac and the neck of intracranial aneurysms including the surrounding vessels. Besides the ability to show opaque vessel structures, the semi-transparent representation is most informative since it conveys important information about the relation between the vessels and the lesion. Above all, the ability of real-time image generation and intuitive manipulation based on the functionality of OpenInventor is most important for a convenient analysis. Since motion enhances the perception of depth, the understanding of complex vessel situations is thereby improved considerably. 
Additionally, integrating shaded vessels obtained by interactive extraction of isovalue surfaces proved to make the understanding of the spatial relation of neighboring objects much easier. Especially, if a fixed direction of view is chosen superimposed structures are inspected more conveniently. Selecting hybrid visualization consisting of a semi-transparent and a iso-surface representation, improved ease of orientation is provided inside cavities or during a user-controlled flight through vessels.

In comparison to other approaches which perform extensive segmentation, the proposed method only provides relative information about the actual vessel wall due to the soft delineation based on transfer functions for color and opacity values. However, the interactivity of the whole visualization process allows a skilled user to change parameters immediately and to evaluate the results by direct visual feedback. As further source of information which ensures the quality of the 3D visualization, clip planes of arbitrary orientation are optionally integrated which provide the original gray values of the data set. As a major advantage of our approach all visualization procedures and the medical diagnosis are performed in less than $15-20$.

The interactive tools which allow to measure distance and volume information directly within the $3 \mathrm{D}$ viewer provide valuable information about the size and the distance of structures. Since clip planes of arbitrary orientation are used for the placement of markers, either the 3D visualization of objects or the original gray value information, optionally shown on every plane, is used to find the correct location. Measurements performed with a phantom data set confirmed the precision of the obtained results.

\section{Conclusion}

A fast and interactive approach was presented for the visualization and analysis of intracranial aneurysms. Using direct volume rendering based on 3D texture mapping and spiral CT angiography, the neck of the aneurysm and the surrounding vessels are optimally conveyed including small aneurysms and complex vessel topology. Improving the perception of depth iso-surface representations are integrated. Based on OpenInventor and communicating windows intuitive manipulation and convenient user-guided navigation are ensured. Further functionality comprises tools for hardware accelerated registration and for volume and distance measurement. The presented results demonstrate the value of our approach which proved to effectively assist pre-operative planning due to the short amount of time required for the complete medical analysis.

\section{References}

1. O. Sommer, A. Dietz, R. Westermann, and T. Ertl. An Interactive Visaulization and Navigation Tool for Medical Volume Data. In V. Skala, editor, WSCG'98 Conf. Proc., Feb. 1998.

2. J.L.D. Atkinson, T.M. Sundt, and O.W. Houser. Angiographic frequency of anterior circulation intracranial aneurysms. Neurosurg., 70:551-555, 1989.

3. S. Nakajima, H. Atsumi, A. Bhalerao, F. Jolesz, R. Kikinis, T. Yoshimine, T. Moriarty, and P. Stieg. Computer-assisted Surgical Planning for Cerebrovascular Neurosurgery. Neurosurgery, 41:403-409, 1997. 
4. K.E.W. Eberhardt, B. Tomandl, W.J. Huk, R. Laumer, and R. Fahlbusch. Value of CT-angiography (CTA) in patients with intracranial aneurysms. Comparison with MRangiography (MRA and digital subtraction angiography (DSA)). In Proc. of 11th Int. Congr. of Neurolog. Surg., pages 1963-1967, Bologna, 1997.

5. A. Puig, D. Tost, and I. Navazo. An Interactive Cerebral Blood Vessel Exploration System. In Proc. Visualization, pages 433-436, Phoenix, AZ, 1997. IEEE Comp. Soc. Press.

6. K.J. Zuiderveld, P.M. van Ooijen, J.W. Chin-A-Woeng, P.C. Buijs, M. Olree, and F.H. Post. Clinical Evaluation of Interactive Volume Visualization. In Proc. Visualization, pages 367370, San Francisco, Calif., 1996. IEEE Comp. Soc. Press.

7. B. Cabral, N. Cam, and J. Foran. Accelerated Volume Rendering and Tomographic Reconstruction Using Texture Mapping Hardware. ACM Symp. on Vol. Vis., pages 91-98, 1994.

8. P. Hastreiter and T. Ertl. Integrated Registration and Visualization of Medical Image Data. In Proc. CGI, pages 78-85, Hannover, Germany, 1998.

9. W. Krüger. The Application of Transport Theory to the Visualization of 3D Scalar Data Fields. In Proc. Visualization, pages 273-280. IEEE Comp. Soc. Press, 1990.

10. J. Danskin and P. Hanrahan. Fast Algorithms for Volume Ray Tracing. In Worksh. of Vol. Vis., pages 91-98. ACM, 1992.

11. R. Yagel and Z. Shi. Accelerating Volume Animation by Space Leaping. In Proc. Visualization, pages 62-69, Los Alamitos, 1993. IEEE Comp. Soc. Press.

12. D. Laur and P. Hanrahan. Hierarchical Splatting: A Progressive Refinement Algorithm for Volume Rendering. Computer Graphics, 25(4):285-288, July 1991.

13. P. Lacroute and M. Levoy. Fast Volume Rendering Using a Shear-Warp Factorization of the Viewing Transform . Computer Graphics, 28(4):451-458, 1994.

14. G. Knittel and W. Straßer. A Compact Volume Rendering Accelerator. In A. Kaufman and W. Krüger, editors, Symp. on Vol. Vis., pages 67-74. ACM SIGGRAPH, 1994.

15. H. Pfister and A. Kaufman. Cube-4 - A Scalable Architecture for Real-Time Volume Rendering. In R. Crawfis and Ch. Hansen, editors, Symp. on Vol. Vis., pages 47-54. ACM SIGGRAPH, 1996.

16. W.E. Lorensen and H.E. Cline. Marching Cubes: A High Resolution 3D Surface Construction Algorithm. Computer Graphics, 21(4):163-169, 1987.

17. J. West and J.M. Fitzpatrick and M.Y. Wang and B.M. Dawant and C.R. Maurer Jr. and R.M. Kessler and R.J. Maciunas. Retrospective Intermodality Registration Techniques: SurfaceBased Versus Volume Based. In Proc. CVRMed-MRCAS, pages 151-160, 1997.

18. A. Collignon, D. Vandermeulen, P. Suetens, and G. Marchal. Automated Multi-Modality Image Registration Based on Information Theory. Kluwen Acad. Publ's: Computational Imaging and Vision, 3:263-274, 1995.

19. W.M. Wells, P. Viola, H. Atsumi, S. Nakajima, and R. Kikinis. Multi-modal Volume Registration by Maximization of Mutual Information. Medical Image Analysis, Oxford University Press, 1(1), March 1996. 

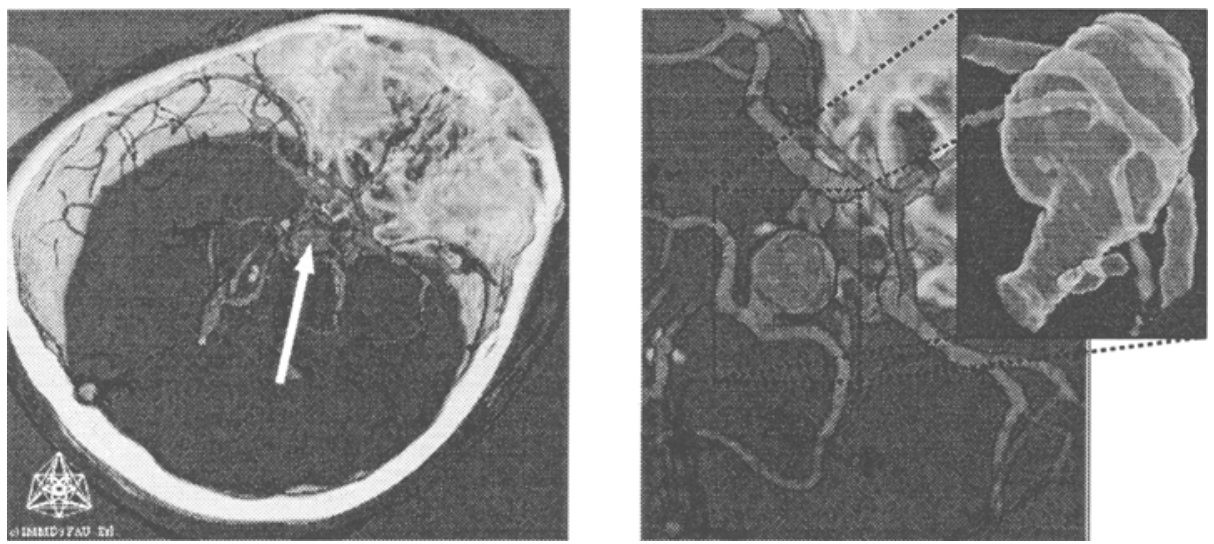

Fig. 6. Basilar bifurcation aneurysm with closer view showing the connected vessels.
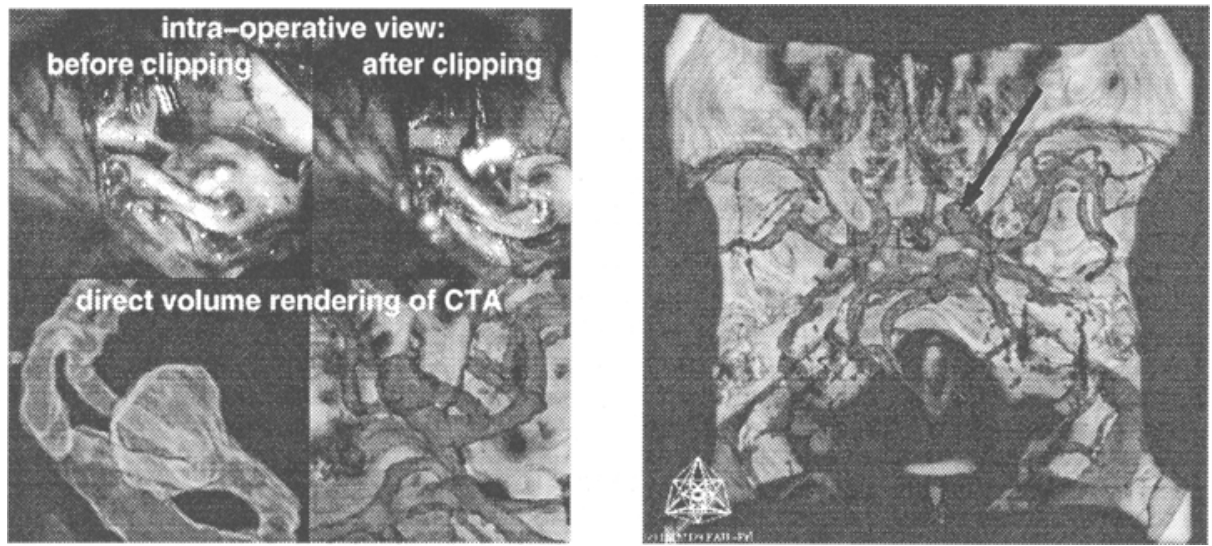

Fig. 7. Aneurysm of anterior communicating artery and comparison with intra-operative views.
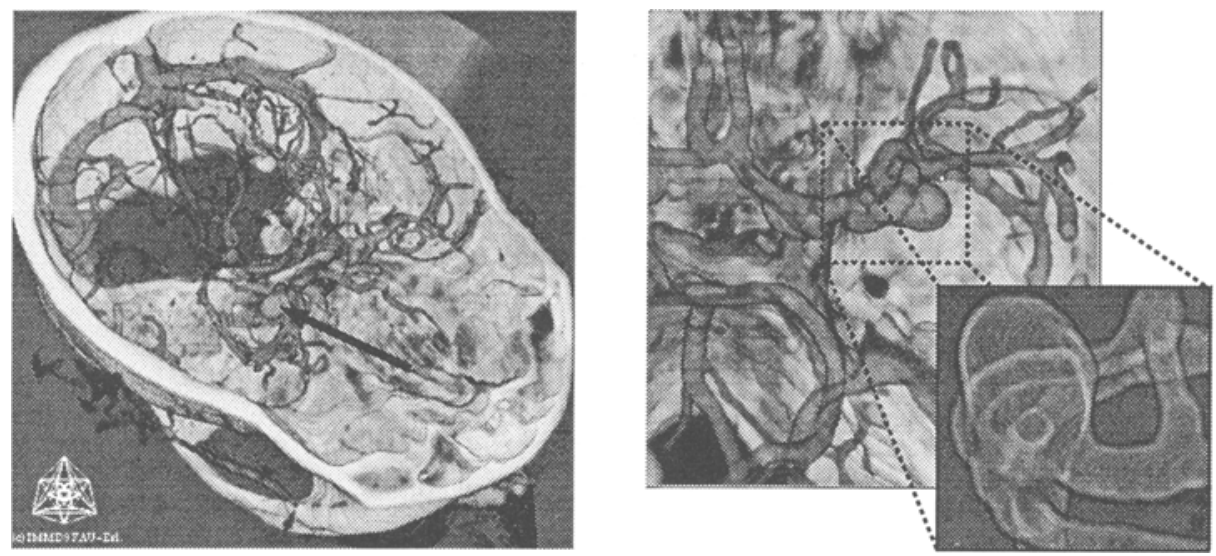

Fig. 8. Aneurysm of middle cerebral artery bifurcation and related vessels. 